\title{
DARBOUX THEORY OF INTEGRABILITY FOR REAL POLYNOMIAL VECTOR FIELDS ON $\mathbb{S}^{n}$
}

\author{
JAUME LLIBRE AND ADRIAN C. MURZA
}

\begin{abstract}
This is a survey on the Darboux theory of integrability for polynomial vector fields, first in $\mathbb{R}^{n}$ and second in the $n$-dimensional sphere $\mathbb{S}^{n}$. We also provide new results about the maximum number of invariant parallels and meridians that a polynomial vector field $\mathcal{X}$ on $\mathbb{S}^{n}$ can have in function of its degree. These results in some sense extend the known result on the maximum number of hyperplanes that a polynomial vector field $\mathcal{Y}$ in $\mathbb{R}^{n}$ can have in function of the degree of $\mathcal{Y}$.
\end{abstract}

\section{Introduction AND StATEMEnt of the main RESUlts}

The real nonlinear ordinary differential systems are used to model a wide range of processes practically all fields of science, from biology and chemistry to economy, physics and engineering. The existence of a first integrals of differential systems defined on $\mathbb{R}^{n}$ is important for two main things. First, they make easier the characterization the phase portrait of the system. Secondly, their existence allow reducing the dimension of the system by one, which in many cases makes easier the analysis. In our terminology, a system is integrable if it has a first integral. Therefore, the methods to detect the presence of first-integrals and find their explicit form are extremely important in the qualitative theory of differential equations.

However, as in many cases occurs, the more important the problem is, the more difficult are the ways to access it. The techniques to finding the presence and constructing first-integrals goes back to Darboux, as far as we know [11]. Hamiltonians are the vector fields whose first-integral are easiest to find. If the integrable vector fields are not Hamiltonian, various techniques have been developed for analyzing the existence of first integrals, such as the Noether symmetries [5], the already mentioned Darbouxian theory of integrability [11] or the Lie symmetries [27]. In fact, Emmy Noether's Theorems represent a relevant example of the interdisciplinary character acquired by the problem of finding first integrals. By (roughly speaking), stating that any physical conservation law has its associated symmetry, it establishes a connection between mechanics, Lie algebra and differential equations. Other contributions to this problem are represented by the Painlevé analysis [3], the use of Lax pairs [17] or the direct method [14] and [15], to cite only a few of them.

2010 Mathematics Subject Classification. Primary: 34C07, 34C05, 34C40.

Key words and phrases. Darboux integrability theory, invariant parallels, invariant meridians, n-dimensional spheres. 
We are especially interested in the Darboux theory of integrability for real polynomial vector fields. This theory provides a method of constructing first-integrals of polynomial vector fields, based on the number of invariant algebraic hypersurfaces that they have. Since its publication in 1878, the method originally developed by Darboux, has been extended and/or refined by many authors both in $\mathbb{R}^{2}$ $[6,7,8,9,11,16,18,25,28,29,30,31,32,33,34]$, and $\mathbb{R}^{n}$ see $[19,20,22,23,24,26]$.

The first objective of this paper is to present the Darboux theory of integrability of the real polynomial vector fields on the $n$-dimensional sphere $\mathbb{S}^{n}=\left\{x \in \mathbb{R}^{2}\right.$ : $\|x\|=1\}$ (here $\|\cdot\|$ denotes the Euclidean norm of $\mathbb{R}^{n+1}$ ), and to study its relation with the maximum number of invariant parallels and meridians that such vector fields can have. Our second objective is to show the kind of parallelism that exist between the Darboux theory of integrability of the real polynomial vector fields on $\mathbb{R}^{n}$ and on $\mathbb{S}^{n}$, and between the invariant hyperplanes of the polynomial vector fields in $\mathbb{R}^{n}$ with the invariant parallels and meridians of the polynomial vector fields on $\mathbb{S}^{n}$.

1.1. Darboux theory of integrability in $\mathbb{R}^{n}$ and invariant hyperplanes. We consider the following polynomial vector field

$$
\mathcal{X}=\sum_{i=1}^{n+1} P_{i}\left(x_{1}, \ldots, x_{n+1}\right) \frac{\partial}{\partial x_{i}}, \quad\left(x_{1}, \ldots, x_{n+1}\right) \in \mathbb{R}^{n+1},
$$

in $\mathbb{R}^{n+1}$, where $P_{i}$ for $i=1, \ldots, n+1$ are polynomials of degree at most $m$. If $m_{i}$ denotes the degree of the polynomial $P_{i}$, we say that $\mathbf{m}=\left(m_{1}, \ldots, m_{d}\right)$ is the degree of the polynomial vector field $\mathcal{X}$. Without loss of generality in the rest of the paper we assume that $m_{1} \geqslant \ldots \geqslant m_{d}$.

Let $\mathbb{C}\left[x_{1}, \ldots, x_{d}\right]$ be the set of all polynomials in the variables $x_{1}, \ldots, x_{n}$ with complex coefficients; in a similar way we define $\mathbb{R}\left[x_{1}, \ldots, x_{d}\right]$. By an invariant algebraic hypersurface of the polynomial vector field $\mathcal{X}$ we understand an algebraic hypersurface $f=f\left(x_{1}, \ldots, x_{d}\right)=0$ with $f \in \mathbb{C}\left[x_{1}, \ldots, x_{d}\right]$ such that for some polynomial $K \in \mathbb{C}\left[x_{1}, \ldots, x_{d}\right]$, we have $\mathcal{X} f=K f$. Moreover, this polynomial $K$ is called the cofactor of the invariant algebraic hypersurface $f=0$. It is worth to noticing that if the degree of the polynomial vector field $\mathcal{X}$ is $\mathbf{m}=\left(m_{1}, \ldots, m_{d}\right)$, with $m_{1} \geq m_{2} \geq \ldots \geq m_{d}$, then any cofactor has at most degree $m_{1}-1$. Moreover, the algebraic hypersurface $f=0$ is called an invariant hyperplane if the degree of $f$ is 1 .

The invariant algebraic hypersurfaces are the "building blocks" for the Darbouxian first-integrals. Their property to separate the phase space of the polynomial vector field $\mathcal{X}$ into invariant pieces makes easier the study of its dynamics.

While $\mathcal{X}$ is a real polynomial vector field, we are working with its complex invariant algebraic hypersurfaces, which in some cases may be real. After stating the next theorem we will rigourously explain this particular situation. For brevity, we remind here that in some cases finding the real first integrals of the real polynomial vector field $\mathcal{X}$ may require the explicit form of the complex invariant algebraic hypersurfaces.

The invariance property of $f$ under the vector field, expressed by $\mathcal{X} f=K f$, leads to the follow remark. Suppose that an orbit of the polynomial vector field $\mathcal{X}$ 
intersects at a point the algebraic hypersurface $f=0$. In that case, the entire orbit is contained in $f=0$. In fact we call $f=0$ flow-invariant, because it is invariant by $\mathcal{X}$. The converse is also true, see [20].

One of the important benefits from refining the Darboux integrability theory concerns the exponential factors. An exponential factor $F\left(x_{1}, \ldots, x_{n}\right)$ of the polynomial vector field $\mathcal{X}$ of degree $m$ is defined as an exponential function of the form $\exp (g / h)$ with $g$ and $h$ polynomials in $\mathbb{C}\left[x_{1}, \ldots, x_{n+1}\right]$ satisfying $\mathcal{X} F=L F$ for some $L \in \mathbb{C}_{m_{1}-1}\left[x_{1}, \ldots, x_{n}\right]$. Here $\mathbb{C}_{m_{1}-1}\left[x_{1}, \ldots, x_{n}\right]$ denotes the set of all polynomials of $\mathbb{C}\left[x_{1}, \ldots, x_{n}\right]$ of degree at most $m_{1}-1$. The exponential factors were introduced by Christopher [7]. They are strongly related to the multiplicity of the invariant algebraic hypersurface $h=0$ if $h$ is not a constant; if $h$ is constant it is related to the multiplicity of the infinity, as discussed in $[10,23,26]$. The fact that an invariant algebraic hypersurface $h=0$ has multiplicity $k$ for a polynomial vector field $\mathcal{X}$, admits the following interpretation. By doing a small perturbation to the vector field $\mathcal{X}$ within the class of polynomial vector fields of the same degree, there are polynomial vector fields $\mathcal{Y}$ near $\mathcal{X}$ having $k$ different invariant algebraic hypersurfaces such that when $\mathcal{Y} \rightarrow \mathcal{X}$ these $k$ hypersurfaces approach to the hypersurface $h=0$.

Let $U \subset \mathbb{R}^{n}$ be an open set. We say that a real function $H\left(x_{1}, \ldots, x_{n}, t\right): \mathbb{R}^{n} \times$ $\mathbb{R} \rightarrow \mathbb{R}$, is an invariant of the polynomial vector field $\mathcal{X}$ on $U$, if $H\left(x_{1}(t), \ldots, x_{n}(t), t\right)$ is constant for all the values of $t$ for which the orbit $\left(x_{1}(t), \ldots, x_{n}(t)\right)$ of $\mathcal{X}$ is contained in $U$.

If the invariant function $H$ is independent on $t$, it is a first integral. If a first integral is a rational function in its variables, then it is called a rational first integral of the vector field $\mathcal{X}$ on $U$.

If the invariant function $H$ is independent on $t$, it is a first integral. We say that a rational first integral of the vector field $\mathcal{X}$ on $U$ if it is a rational function in its variables.

In the next theorem we summarize the Darboux theory for polynomial vector fields in $\mathbb{R}^{n}$ which has been extended to the Darboux theory for polynomial vector fields in $\mathbb{S}^{n}$.

Theorem 1. Suppose that a polynomial vector field $\mathcal{X}$ defined in $\mathbb{R}^{n}$ of degree $m=\left(m_{1}, \ldots, m_{n}\right)$ admits $p$ invariant algebraic hypersurfaces $f_{i}=0$ with cofactors $K_{i}$ for $i=1, \ldots, p$, and $q$ exponential factors $F_{j}=\exp \left(g_{j} / h_{j}\right)$ with cofactors $L_{j}$ for $j=1, \ldots, q$. Then the following statements hold.

(a) There exist $\lambda_{i}, \mu_{j} \in \mathbb{C}$ not all zero such that $\sum_{i=1}^{p} \lambda_{i} K_{i}+\sum_{j=1}^{q} \mu_{j} L_{j}=0$, if and only if the real (multi-valued) function of Darbouxian type

$$
f_{1}^{\lambda_{1}} \ldots f_{p}^{\lambda_{p}} F_{1}^{\mu_{1}} \ldots F_{q}^{\mu_{q}}
$$

substituting $f_{i}^{\lambda_{i}}$ by $\left|f_{i}\right|^{\lambda_{i}}$ if $\lambda_{i} \in \mathbb{R}$, is a first integral of the vector field $\mathcal{X}$. 
(b) If $p+q \geqslant\left(\begin{array}{c}n+m_{1}-1 \\ m_{1}-1\end{array}\right)+1$, then there exist $\lambda_{i}, \mu_{j} \in \mathbb{C}$ not all zero such that $\sum_{i=1}^{p} \lambda_{i} K_{i}+\sum_{j=1}^{q} \mu_{j} L_{j}=0$.

(c) There exist $\lambda_{i}, \mu_{j} \in \mathbb{C}$ not all zero such that $\sum_{i=1}^{p} \lambda_{i} K_{i}+\sum_{j=1}^{q} \mu_{j} L_{j}=-\sigma$ for some $\sigma \in \mathbb{R} \backslash\{0\}$, if and only if the real (multi-valued) function

$$
f_{1}^{\lambda_{1}} \ldots f_{p}^{\lambda_{p}} F_{1}^{\mu_{1}} \ldots F_{q}^{\mu_{q}} e^{\sigma t}
$$

substituting $f_{i}^{\lambda_{i}}$ by $\left|f_{i}\right|^{\lambda_{i}}$ if $\lambda_{i} \in \mathbb{R}$, is an invariant of the vector field $\mathcal{X}$.

(d) The vector field $\mathcal{X}$ has a rational first integral if and only if

$$
p+q \geqslant\left(\begin{array}{c}
n+m_{1}-1 \\
m_{1}-1
\end{array}\right)+n .
$$

Moreover, all trajectories are contained in invariant algebraic hypersurfaces.

For a proof of Theorem 1 see for instance [13, 23, 24, 25].

In the statement of the Darboux theory of integrability for polynomial vector fields in $\mathbb{R}^{n}$ given in Theorem 1 we only have taken into account partially the multiplicity of the invariant algebraic hypersurfaces $f_{i}=0$ through the existence of integrating factors, for more details see [10] and [23].

In statement (a) of Theorem 1 we said that the function (1) is real. This is due to the following fact. Since the vector field $\mathcal{X}$ is real, it is well known that if a complex invariant algebraic hypersurface or exponential factor appears, then its conjugate has to appear simultaneously. If among the invariant algebraic hypersurfaces of $\mathcal{X}$ a complex conjugate pair $f=0$ and $\bar{f}=0$ occur, then the first integral (1) has a real factor of the form $f^{\lambda} \bar{f}^{\bar{\lambda}}$, which is the multi-valued real function

$$
\left[(\operatorname{Re} f)^{2}+(\operatorname{Im} f)^{2}\right]^{\operatorname{Re} \lambda} \exp \left(-2 \operatorname{Im} \lambda \arctan \left(\frac{\operatorname{Im} f}{\operatorname{Re} f}\right)\right),
$$

if $\operatorname{Im} \lambda \operatorname{Im} f \not \equiv 0$. If among the exponential factors of $\mathcal{X}$ a complex conjugate pair $F=\exp (h / g)$ and $\bar{F}=\exp (\bar{h} / \bar{g})$ occur, the first integral (1) has a real factor of the form

$$
\left(\exp \left(\frac{h}{g}\right)\right)^{\mu}\left(\exp \left(\frac{\bar{h}}{\bar{g}}\right)\right)^{\bar{\mu}}=\exp \left(2 \operatorname{Re}\left(\mu \frac{h}{g}\right)\right)
$$

One of the best tools for searching for invariant algebraic hypersurfaces is the extactic polynomial of $\mathcal{X}$ associated to $W$. To our knowledge it was first mentioned in the work of Lagutinskii, see [35]. To define it let $W$ be a finitely generated vector subspace of vector space $\mathbb{C}\left[x_{1}, \ldots, x_{d}\right]$. The extactic polynomial of $\mathcal{X}$ associated to $W$ is

$$
\mathcal{E}_{W}=\mathcal{E}_{\left\{v_{1}, \ldots, v_{l}\right\}}(\mathcal{X})=\operatorname{det}\left(\begin{array}{cccc}
v_{1} & v_{2} & \ldots & v_{l} \\
\mathcal{X}\left(v_{1}\right) & \mathcal{X}\left(v_{2}\right) & \ldots & \mathcal{X}\left(v_{l}\right) \\
\vdots & \vdots & \ldots & \vdots \\
\mathcal{X}^{l-1}\left(v_{1}\right) & \mathcal{X}^{l-1}\left(v_{2}\right) & \ldots & \mathcal{X}^{l-1}\left(v_{l}\right)
\end{array}\right)=0,
$$


where $\left\{v_{1}, \ldots, v_{l}\right\}$ is a basis of $W, l$ is the dimension of $W$, and $\mathcal{X}^{j}\left(v_{i}\right)=$ $\mathcal{X}^{j-1}\left(\mathcal{X}\left(v_{i}\right)\right)$. It is well known that one of the main properties of the extactic polynomial is that its definition does not dependent of the chosen basis of $W$.

In this paper the reason to using the extactic polynomial $\mathcal{E}_{W}(\mathcal{X})$ is twofold. Firstly, it allows detecting algebraic hypersurfaces $f=0$ with $f \in W$ such that they are invariant by the polynomial vector field $\mathcal{X}$, see the following proposition proved in [10]. Secondly, it allows to defining and computing the multiplicity of invariant algebraic hypersurfaces.

Even if the next proposition is stated for complex polynomial vector fields, it is very useful for our later considerations. This is so, because we deal with real polynomial vector fields, which are particular cases of complex ones.

Proposition 2. Let $\mathcal{X}$ be a polynomial vector field in $\mathbb{C}^{d}$ and let $W$ be a finitely generated vector subspace of $\mathbb{C}\left[x_{1}, \ldots, x_{d}\right]$ with $\operatorname{dim}(W)>1$. Then every algebraic invariant hypersurface $f=0$ for the vector field $\mathcal{X}$, with $f \in W$, is a factor of the polynomial $\mathcal{E}_{W}(\mathcal{X})$.

From Proposition 2 it follows that $f=0$ is an invariant hyperplane of the polynomial vector field $\mathcal{X}$ if the polynomial $f$ is a factor of the polynomial $\mathcal{E}_{W}(\mathcal{X})$, where $W$ is generated by $\left\{1, x_{1}, \ldots, x_{d}\right\}$. From [10] the invariant hyperplane $f=0$ has multiplicity $k$ if $k$ is the greatest positive integer such that $f^{k}$ divides the polynomial $\mathcal{E}_{W}(\mathcal{X})$

An important result on the maximum number of invariant hyperplanes that a polynomial vector field in $\mathbb{R}^{n}$ can have was proved in [20]. The analogous result for polynomial vector fields in $\mathbb{R}^{2}$ was proved before in [2].

Theorem 3. Assume that a polynomial vector field $\mathcal{X}$ in $\mathbb{R}^{n}$ or $\mathbb{C}^{n}$ with $n \geq 2$ of degree $\mathbf{m}=\left(m_{1}, \ldots, m_{d}\right)$ with $m_{1} \geq \ldots \geq m_{n}$ has finitely many invariant hyperplanes. Then

(1) the number of invariant hyperplanes of $\mathcal{X}$ taking into account their multiplicities is at most

$$
\left(\begin{array}{c}
n \\
2
\end{array}\right)\left(m_{1}-1\right)+\left(\sum_{k=1}^{n} m_{k}\right)
$$

(2) the number of invariant hyperplanes of $\mathcal{X}$ through a single point taking into account their multiplicities taking into account their multiplicities is at most

$$
\left(\begin{array}{c}
n-1 \\
2
\end{array}\right)\left(m_{1}-1\right)+\left(\sum_{k=1}^{n-1} m_{k}\right)+1 .
$$

Moreover there are complex polynomial vector fields which reach this upper bound for their number of invariant hyperplanes taking into account their multiplicities.

Remark 4. Knowing a sufficient number of invariant hyperplanes such that their cofactors satisfy the assumptions either of statement (a) or of statement (b) of Theorem 1 enable us to find a first integral of the polynomial vector field $\mathcal{X}$. 
1.2. Darboux theory of integrability in $\mathbb{S}^{n}$ and invariant parallels and meridians. Before stating our results, we need a few preliminary definitions for hypersurfaces we are dealing with. First we give the general introductory notions valid for the application of the Darboux theory of integrability on any smooth hypersurfaces; then we will focus on the $n$-dimensional sphere $\mathbb{S}^{n}$ and its invariant meridians and parallels.

Let $G: \mathbb{R}^{n+1} \rightarrow \mathbb{R}$ be a $C^{1}$ map. A hypersurface $\boldsymbol{\Omega}=\left\{\left(x_{1}, \ldots, x_{n+1}\right) \in \mathbb{R}^{n+1}\right.$ : $\left.G\left(x_{1}, \ldots, x_{n+1}\right)=0\right\}$ is said to be regular if the gradient $\nabla G$ of $G$ is not equal to zero on $\Omega$. Of course, if $\Omega$ is regular, then it is smooth. We say that $\Omega$ is algebraic if $G$ is an irreducible polynomial. If the degree of the polynomial $G$ is $d$, then we say that $\Omega$ is algebraic of degree $d$. A polynomial vector field $\mathcal{X}$ on the regular hypersurface $\Omega$ (or simply a polynomial vector field on $\Omega$ ) is a polynomial vector field $\mathcal{X}$ in $\mathbb{R}^{n+1}$ satisfying

$$
\left(P_{1}, \ldots, P_{n+1}\right) \cdot \nabla G=0 \text { on the points of } \Omega,
$$

where the dot denotes the inner product of two vectors in $\mathbb{R}^{n+1}$. If the polynomial vector field $\mathcal{X}$ in $\mathbb{R}^{n+1}$ has degree $m$, then we say that the vector field $\mathcal{X}$ on $\Omega$ is of degree $m$.

Let $f=f\left(x_{1}, \ldots, x_{n+1}\right) \in \mathbb{C}\left[x_{1}, \ldots, x_{n+1}\right]$. We say that the algebraic hypersurface $\{f=0\} \cap \Omega \subset \mathbb{R}^{n+1}$ is invariant by the polynomial vector field $\mathcal{X}$ on $\Omega$ (or simply an invariant algebraic hypersurface on $\Omega$ ) if it satisfies

(i) there exists a polynomial $k \in \mathbb{C}\left[x_{1}, \ldots, x_{n+1}\right]$ such that

$$
\mathcal{X} f=\sum_{i=1}^{n+1} P_{i} \frac{\partial f}{\partial x_{i}}=k f \text { on } \Omega,
$$

the polynomial $k=k\left(x_{1}, \ldots, x_{n+1}\right) \in \mathbb{C}\left[x_{1}, \ldots, x_{n+1}\right]$ is called the cofactor of $f=0$ on $\Omega$;

(ii) the two hypersurfaces $f=0$ and $\Omega$ have transversal intersection, i.e. if the vectors $\nabla G$ and $\nabla f$ are independent in all the points of the hypersurface $\{f=0\} \cap \Omega$.

Clearly the vector field $\mathcal{X}$ is tangent to the algebraic hypersurface $\{f=0\} \cap \Omega$. So the hypersurface $\{f=0\} \cap \Omega$ is formed by orbits of the vector field $\mathcal{X}$. This explains why we say that the algebraic hypersurface $\{f=0\} \cap \Omega$ is invariant by the flow of the vector field $\mathcal{X}$.

An exponential factor $F=F\left(x_{1}, \ldots, x_{n+1}\right)$ of the polynomial vector field $\mathcal{X}$ of degree $m$ on the regular hypersurface $\Omega$ is an exponential function of the form $\exp (g / h)$ with $g$ and $h$ polynomials in $\mathbb{C}\left[x_{1}, \ldots, x_{n+1}\right]$ and satisfying $\mathcal{X} F=L F$ on $\Omega$ for some $K \in \mathbb{C}_{m-1}\left[x_{1}, \ldots, x_{n+1}\right]$.

Let $f$ and $g$ be two polynomials of $\mathbb{C}_{m}\left[x_{1}, x_{2}, \ldots, x_{n+1}\right]$ and let $\Omega=\{G=0\}$ be a smooth algebraic hypersurface in $\mathbb{R}^{n+1}$ of degree $d$. We say that $f$ and $g$ are related, $f \sim g$, if either $f / g=$ constant or $f-g=h G$ for some polynomial $h$. That is, $\sim$ is an equivalence relation in $\mathbb{C}_{m}\left[x_{1}, x_{2}, \ldots, x_{n+1}\right]$; it partitions $\mathbb{C}_{m}\left[x_{1}, x_{2}, \ldots, x_{n+1}\right]$ into equivalence classes defined in the following way. Given a set $\mathbb{C}_{m}\left[x_{1}, x_{2}, \ldots, x_{n+1}\right]$ and the equivalence relation $\sim$ on $\mathbb{C}_{m}\left[x_{1}, x_{2}, \ldots, x_{n+1}\right]$, the equivalence class of an 
element $g$ in $\mathbb{C}_{m}\left[x_{1}, x_{2}, \ldots, x_{n+1}\right]$ is the set

$$
\left\{f \in \mathbb{C}_{m}\left[x_{1}, x_{2}, \ldots, x_{n+1}\right] \mid f \sim g\right\} .
$$

In both cases mentioned in this paragraph $\Omega \cap\{f=0\}=\Omega \cap\{g=0\}$. The result of the partition of $\mathbb{C}_{m}\left[x_{1}, x_{2}, \ldots, x_{n+1}\right]$ by the equivalence relation $\sim$ into equivalence classes yields the quotient space $\mathbb{C}_{m}\left[x_{1}, \ldots, x_{n+1}\right] / \sim$; we denote its dimension by $d(m)$, called the dimension of $\mathbb{C}_{m}\left[x_{1}, \ldots, x_{n+1}\right]$ on $\Omega$. In [22] it is proved that the dimension of $\mathbb{C}_{m}\left[x_{1}, \ldots, x_{n}\right] / \sim$ is

$$
d(m)=\left(\begin{array}{c}
n+m \\
n
\end{array}\right)-\left(\begin{array}{c}
n+m-d \\
n
\end{array}\right) .
$$

Let $U \subset \mathbb{R}^{n+1}$ be an open set. A real function $H\left(x_{1}, \ldots, x_{n+1}, t\right): \mathbb{R}^{n+1} \times$ $\mathbb{R} \rightarrow \mathbb{R}$, is said to be invariant of the polynomial vector field $\mathcal{X}$ on $\Omega \cap U$, if $H\left(x_{1}(t), \ldots, x_{n+1}(t), t\right)=$ constant for all the values of $t$ for which the orbit $\left(x_{1}(t), \ldots, x_{n+1}(t)\right)$ of $\mathcal{X}$ is contained in $\Omega \cap U$.

If an invariant $H$ is independent on $t$, then $H$ is a first integral. If a first integral $H$ is a rational function in its variables, then it is called a rational first integral of the vector field $\mathcal{X}$ on $\Omega \cap U$.

Now we present the extension of the Darboux theory of integrability to polynomial vector fields on $\mathbb{S}^{n}$.

Theorem 5. Assume that $\mathcal{X}$ is a polynomial vector field on $\mathbb{S}^{n}$ of degree $m=$ $\left(m_{1}, \ldots, m_{n}\right)$ having $p$ invariant algebraic hypersurfaces $\left\{f_{i}=0\right\} \cap \mathbb{S}^{n}$ with cofactors $K_{i}$ for $i=1, \cdots, p$, and $q$ exponential factors $F_{j}=\exp \left(g_{j} / h_{j}\right)$ with cofactors $L_{j}$ for $j=1, \cdots, q$. Then the following statements hold.

(a) There exist $\lambda_{i}, \mu_{j} \in \mathbb{C}$ not all zero such that $\sum_{i=1}^{p} \lambda_{i} K_{i}+\sum_{j=1}^{q} \mu_{j} L_{j}=0$ on $\mathbb{S}^{n}$, if and only if the real (multi-valued) function of Darbouxian type

$$
f_{1}^{\lambda_{1}} \cdots f_{p}^{\lambda_{p}} F_{1}^{\mu_{1}} \cdots F_{q}^{\mu_{q}},
$$

substituting $f_{i}^{\lambda_{i}}$ by $\left|f_{i}\right|^{\lambda_{i}}$ if $\lambda_{i} \in \mathbb{R}$, is a first integral of the vector field $\mathcal{X}$ on $\mathbb{S}^{n}$.

(b) If $p+q \geqslant \frac{n+2 m_{1}}{n+m_{1}}\left(\begin{array}{c}n+m_{1} \\ m_{1}\end{array}\right)+1$, then there exist $\lambda_{i}, \mu_{j} \in \mathbb{C}$ not all zero such that $\sum_{i=1}^{p} \lambda_{i} K_{i}+\sum_{j=1}^{q} \mu_{j} L_{j}=0$ on $\mathbb{S}^{n}$.

(c) There exist $\lambda_{i}, \mu_{j} \in \mathbb{C}$ not all zero such that $\sum_{i=1}^{p} \lambda_{i} K_{i}+\sum_{j=1}^{q} \mu_{j} L_{j}=-\sigma$ on $\mathbb{S}^{n}$ for some $\sigma \in \mathbb{R} \backslash\{0\}$, if and only if the real (multi-valued) function

$$
f_{1}^{\lambda_{1}} \cdots f_{p}^{\lambda_{p}} F_{1}^{\mu_{1}} \cdots F_{q}^{\mu_{q}} e^{\sigma t},
$$

substituting $f_{i}^{\lambda_{i}}$ by $\left|f_{i}\right|^{\lambda_{i}}$ if $\lambda_{i} \in \mathbb{R}$, is an invariant of $\mathcal{X}$ on $\mathbb{S}^{n}$. 
(d) The vector field $\mathcal{X}$ on $\mathbb{S}^{n}$ has a rational first integral if and only if $p+$ $q \geqslant \frac{n+2 m_{1}}{n+m_{1}}\left(\begin{array}{c}n+m_{1} \\ m_{1}\end{array}\right)+n$. Moreover, all trajectories are contained in invariant algebraic hypersurfaces.

The proof of statements (a), (b) and (c) of Theorem 5 was done in [22], and the proof of its statement $(d)$ in [19].

The parallels of the $n$-dimensional sphere $\mathbb{S}^{n}$ are the intersections of the hyperplanes $x_{n+1}=$ constant with the sphere $\mathbb{S}^{n}$, and the meridians are the intersections of the hyperplanes containing the $x_{n+1}$-axis with the sphere $\mathbb{S}^{n}$. Now we need to extend Theorem 3 to the parallels and meridians.

In the next theorem we provide the maximum number of invariant meridians that a polynomial vector field in $\mathbb{S}^{n}$ can have in function of its degree.

Theorem 6. For $n \geqslant 2$ let $\mathcal{X}$ be a polynomial vector field on $\mathbb{S}^{n}$ of degree $\mathbf{m}=$ $\left(m_{1}, \ldots, m_{n+1}\right)$ with $m_{1} \geqslant m_{2} \geqslant \ldots \geqslant m_{n+1}$. Assume that $\mathcal{X}$ has finitely many invariant meridians. Then the number of invariant meridians of $\mathcal{X}$ is at most

$$
\left(\begin{array}{c}
n-1 \\
2
\end{array}\right)\left(m_{1}-1\right)+\left(\sum_{i=1}^{n-1} m_{i}\right)+1
$$

where $\left(\begin{array}{c}n-1 \\ 2\end{array}\right)=0$ if $n=2$.

Proof. By definition an invariant meridian of $\mathbb{S}^{n}$ by the vector field $\mathcal{X}$ is obtained intersecting an invariant hyperplane of $\mathcal{X}$ of the form $\sum_{i=1}^{n} a_{i} x_{i}=0$ (which contain the $x_{n+1}$-axis) with $\mathbb{S}^{n}$. So in order to determine an upper bound for the number of invariant meridians of $\mathcal{X}$ it suffices to determine an upper bound for the number of invariant hyperplanes $\sum_{i=1}^{n} a_{i} x_{i}=0$ of $\mathcal{X}$.

By Proposition 2 if $\sum_{i=1}^{n} a_{i} x_{i}=0$ is an invariant hyperplane of $\mathcal{X}$ it must divide the extactic polynomial $\mathcal{E}_{\left\{x_{1} \ldots, x_{n}\right\}}(\mathcal{X})$. Therefore the degree of this extactic polynomial is an upper bound for the maximal number of meridians.

From the definition of extactic polynomial we have

$$
\mathcal{E}_{\left\{x_{1}, \ldots, x_{n}\right\}}(\mathcal{X})=\left|\begin{array}{cccccc}
x_{1} & x_{2} & x_{3} & \ldots & x_{n-1} & x_{n} \\
P_{1} & P_{2} & P_{3} & \ldots & P_{n-1} & P_{n} \\
\mathcal{X}\left(P_{1}\right) & \mathcal{X}\left(P_{2}\right) & \mathcal{X}\left(P_{3}\right) & \ldots & \mathcal{X}\left(P_{n-1}\right) & \mathcal{X}\left(P_{n}\right) \\
\vdots & \vdots & \vdots & \vdots & \vdots & \\
\mathcal{X}^{n-4}\left(P_{1}\right) & \mathcal{X}^{n-4}\left(P_{2}\right) & \mathcal{X}^{n-4}\left(P_{3}\right) & \cdots & \mathcal{X}^{n-4}\left(P_{n-1}\right) & \mathcal{X}^{n-4}\left(P_{n}\right) \\
\mathcal{X}^{n-3}\left(P_{1}\right) & \mathcal{X}^{n-3}\left(P_{2}\right) & \mathcal{X}^{n-3}\left(P_{3}\right) & \ldots & \mathcal{X}^{n-3}\left(P_{n-1}\right) & \mathcal{X}^{n-3}\left(P_{n}\right) \\
\mathcal{X}^{n-2}\left(P_{1}\right) & \mathcal{X}^{n-2}\left(P_{2}\right) & \mathcal{X}^{n-2}\left(P_{3}\right) & \ldots & \mathcal{X}^{n-2}\left(P_{n-1}\right) & \mathcal{X}^{n-2}\left(P_{n}\right)
\end{array}\right|
$$


Since $m_{1} \geqslant m_{2} \geqslant m_{3} \geqslant \ldots \geqslant m_{n+1}$, we have that the degree of the extactic polynomial $\mathcal{E}_{\left\{x_{1}, \ldots, x_{n}\right\}}(\mathcal{X})$ is at most the degree of the polynomial

$$
\mathcal{P}=\mathcal{X}^{n-2}\left(P_{1}\right) \mathcal{X}^{n-3}\left(P_{2}\right) \mathcal{X}^{n-4}\left(P_{3}\right) \cdots \mathcal{X}\left(P_{n-2}\right) P_{n-1} x_{n} .
$$

Clearly the degree of $\mathcal{X}^{\ell}\left(P_{i}\right)$ is $\ell\left(m_{1}-1\right)+m_{i}$, so the degree of $\mathcal{P}$ is

$$
\begin{aligned}
\operatorname{deg}(\mathcal{P})= & \left((n-2)\left(m_{1}-1\right)+m_{1}\right)+\left((n-3)\left(m_{1}-1\right)+m_{2}\right) \\
& +\cdots+\left(\left(m_{1}-1\right)+m_{n-2}\right)+m_{n-1}+1 .
\end{aligned}
$$

If $n>2$ then

$$
\begin{aligned}
\operatorname{deg}(\mathcal{P} & =\left(\sum_{k=1}^{n-2} k\right)\left(m_{1}-1\right)+\left(\sum_{k=1}^{n-1} m_{k}\right)+1 \\
& =\left(\begin{array}{c}
n-1 \\
2
\end{array}\right)\left(m_{1}-1\right)+\left(\sum_{i=1}^{n-1} m_{i}\right)+1
\end{aligned}
$$

If $n=2$ then

$$
\operatorname{deg}(\mathcal{P})=m_{1}+1 .
$$

This completes the proof of the theorem.

In the next theorem we provide the maximum number of invariant parallels that a polynomial vector field in $\mathbb{S}^{n}$ can have in function of its degree.

Theorem 7. Let $\mathcal{X}$ be a polynomial vector field on $\mathbb{S}^{n}$ of degree $\mathbf{m}=\left(m_{1}, \ldots, m_{n+1}\right)$ with $m_{1} \geqslant m_{2} \geqslant \ldots \geqslant m_{n+1}$. Assume that $\mathcal{X}$ has finitely many invariant parallels. Then the number of invariant parallels of $\mathcal{X}$ is at most $m_{n+1}$.

Proof. By definition an invariant parallel is the intersection of an invariant hyperplane of the form $x_{n+1}=k$, where $k \in(-1,1)$, with the sphere $\mathbb{S}^{n}$. Thus this intersection is an $\mathbb{S}^{n-1}$ sphere of radius $\sqrt{1-k^{2}}$.

From Proposition 2 we know that if $x_{n+1}-k=0$ is an invariant hyperplane of polynomial vector field $\mathcal{X}$, then $x_{n+1}-k$ is a factor of the extactic polynomial

$$
\mathcal{E}_{\left\{1, x_{n+1}\right\}}(\mathcal{X})=\left|\begin{array}{cc}
1 & x_{n+1} \\
\mathcal{X}(1) & \mathcal{X}\left(x_{n+1}\right)
\end{array}\right|=\left|\begin{array}{cc}
1 & x_{n+1} \\
0 & \dot{x}_{n+1}
\end{array}\right|=\dot{x}_{n+1}=P_{n+1}\left(x_{1}, \ldots, x_{n+1}\right) .
$$

Since the degree of $P_{n+1}$ is $m_{n+1}$, this polynomial at most can have $m_{n+1}$ linear factors of the form $x_{n+1}-k$. Hence it follows that the number of invariant parallels of $\mathcal{X}$ is at most $m_{n+1}$.

Next we will prove that if the polynomial vector field $\mathcal{X}$ on $\mathbb{S}^{2}$ of degree $\mathbf{m}=$ $\left(m_{1}, m_{2}\right)$ with $m_{1} \geqslant m_{2}$ is complex, then it can reach the maximum upper bound $m_{1}+1$ for the number of invariant meridians stated in Theorem 6 , but if $\mathcal{X}$ is real then we have examples with $m_{1}$ invariant meridians. This result that for real polynomial vector fields the upper bounds of some invariant objects cannot be reached, but they can be reached for the complex polynomial vector fields already occurred for polynomial vector field $\mathcal{X}$ in the plane. Thus in the complex plane $\mathbb{C}^{2}$ a polynomial vector field $\mathcal{X}$ of degree $\mathbf{m}=\left(m_{1}, m_{2}\right)$ can have at most $3 m_{1}-1$ invariant straight lines, and this bound is reached, but if $\mathcal{X}$ is in $\mathbb{R}^{2}$ we only know that this bound is in the interval $\left[2 m_{1}+1,3 m_{1}-1\right]$ if $m_{1}$ is even and $\left[2 m_{1}+2,3 m_{1}-1\right]$ if $m_{1}$ is odd, see for more details [2,10]. 
In the following proposition we provide a complex polynomial vector field $\mathcal{X}$ of degree $(2,2)$ on the complex sphere $\mathbb{S}^{2}$ where the upper bound $m_{1}+1=3$ for the maximum number of invariant meridians is reached, and we prove that the real polynomial vector field $\mathcal{X}$ of degree $\mathbf{m}=(2,2)$ on the real sphere $\mathbb{S}^{2}$ cannot have $m_{1}+1=3$ invariant meridians. Additionally we provide a real polynomial vector field $\mathcal{X}$ of degree $(2,2)$ on the real sphere $\mathbb{S}^{2}$ with $m_{1}=2$ invariant meridians.

Proposition 8. The following statements hold.

(a) The complex polynomial differential system

$\dot{x}=i y(x+y)-2 x z, \quad \dot{y}=-i x(x+y)-2 y z, \quad \dot{z}=1+x^{2}+y^{2}-z^{2}$,

on the complex sphere $\mathbb{S}^{2}$ of degree $(2,2,2)$ has the maximum number of complex invariant meridians $m_{1}+1=3$, namely $x+i y=0, x-i y=0$ and $x+y+1=0$.

(b) There are no real polynomial differential systems on the real sphere $\mathbb{S}^{2}$ of degree $(2,2,2)$ realizing the maximum number of real invariant meridians $m_{1}+1=3$. Such polynomial differential systems have at most 2 real invariant meridians, and this upper bound is reached.

Proof. Consider the vector field $\mathcal{X}$ associated to the complex polynomial differential system of statement $(a)$. Then

$$
\mathcal{X}\left(x^{2}+y^{2}+z^{2}-1\right)=-2 z\left(x^{2}+y^{2}+z^{2}-1\right),
$$

so $\mathcal{X}$ is a polynomial vector field on the complex sphere $\mathbb{S}^{2}$. The three complex planes $x+i y=0, x-i y=0$ and $x+y+1=0$ are invariant because

$$
\begin{aligned}
& \mathcal{X}(x+i y)=(x+y-2 z)(x+i y), \\
& \mathcal{X}(x-i y)=-(x+y+2 z)(x-i y), \\
& \mathcal{X}(x+y)=-i(x-y-2 i z)(x+y) .
\end{aligned}
$$

This completes the proof of statement $(a)$.

Now we shall prove statement $(b)$. Consider a general real polynomial differential system of degree $(2,2,2)$ in $\mathbb{R}^{3}$, i.e.

$$
\dot{x}=\sum_{i+j+k=0}^{2} a_{i j k} x^{i} y^{j} z^{k}, \quad \dot{y}=\sum_{i+j+k=0}^{2} b_{i j k} x^{i} y^{j} z^{k}, \quad \dot{z}=\sum_{i+j+k=0}^{2} c_{i j k} x^{i} y^{j} z^{k} .
$$

Imposing that $\mathcal{X}\left(x^{2}+y^{2}+z^{2}-1\right)=\left(k_{0}+k_{1} x+k_{2} y+k_{3} z\right)\left(x^{2}+y^{2}+z^{2}-1\right)$ system (3) becomes

$$
\begin{aligned}
\dot{x}= & a_{000}-b_{100} y-c_{100} z-a_{000} x^{2}-\left(b_{000}+b_{200}\right) x y-\left(c_{000}+c_{200}\right) x z \\
& -\left(a_{000}+b_{110}\right) y^{2}-\left(b_{101}+c_{110}\right) y z-\left(a_{000}+c_{101}\right) z^{2} \\
\dot{y}= & b_{000}+b_{100} x+b_{200} x^{2}+b_{110} x y-b_{000} y^{2}-c_{010} z+b_{101} x z \\
& -\left(c_{000}+c_{020}\right) y z-\left(b_{000}+c_{011}\right) z^{2}, \\
\dot{z}= & c_{000}+c_{100} x+c_{200} x^{2}+c_{010} y+c_{110} x y+c_{020} y^{2}+c_{101} x z \\
& +c_{011} y z-c_{000} z^{2} .
\end{aligned}
$$

Now we impose that the plane $a x+b y=0$ be invariant by the polynomial differential system $(4)$, i.e. $\mathcal{X}(a+b y)=\left(k_{0}+k_{1} x+k_{2} y+k_{3} z\right)(a x+b y)$ and we 
obtain three possible polynomial differential systems (4) having invariant planes of this kind.

First the polynomial differential system

$$
\begin{aligned}
\dot{x}= & a_{000}-c_{100} z-a_{000} x^{2}-\left(c_{000}+c_{200}\right) x z-\left(a_{000}+b_{110}\right) y^{2} \\
& -c_{110} y z-\left(a_{000}+c_{101}\right) z^{2}, \\
\dot{y}= & b_{110} x y-\left(c_{000}+c_{020}\right) y z, \\
\dot{z}= & c_{000}+c_{100} x+c_{200} x^{2}+c_{110} x y+c_{020} y^{2}+c_{101} x z-c_{000} z^{2},
\end{aligned}
$$

having the invariant plane $y=0$, and at most it has another invariant plane of the form $a x+b y=0$, namely $x=0$, because its extactic polynomial is

$y\left(b_{110}\left(x^{2}+y^{2}\right)+z\left(c_{100}-c_{020} x+c_{200} x+c_{110} y+c_{101} z\right)+a_{000}\left(-1+x^{2}+y^{2}+z^{2}\right)\right)$,

and taking $b_{110}=c_{100}=c_{110}=c_{101}=a_{000}=0$ it is easy to check that it has also the invariant plane $x=0$.

Second the polynomial differential system

$$
\begin{aligned}
\dot{x}= & -\left(b_{000}+b_{200}\right) x y-\left(c_{000}+c_{200}\right) x z \\
\dot{y}= & b_{000}-c_{010} z+b_{200} x^{2}-b_{000} y^{2}+b_{101} x z-\left(c_{000}+c_{020}\right) y z \\
& -\left(b_{000}+c_{011}\right) z^{2} \\
\dot{z}= & c_{000}+c_{010} y+c_{200} x^{2}-b_{101} x y+c_{020} y^{2}+c_{011} y z-c_{000} z^{2},
\end{aligned}
$$

having the invariant plane $x=0$, and at most it has another invariant plane of the form $a x+b y=0$, namely $y=0$, because its extactic polynomial is

$$
x\left(b_{000}+b_{200}\left(x^{2}+y^{2}\right)+\left(-c_{010}+b_{101} x+\left(-c_{020}+c_{200}\right) y\right) z-\left(b_{000}+c_{011}\right) z^{2}\right) ;
$$

and taking $b_{000}=b_{200}=c_{010}=b_{101}=c_{011}=0$ it is easy to check that it has also the invariant plane $y=0$.

Finally the polynomial differential system

$$
\begin{aligned}
\dot{x}= & -b b_{000} / a-c_{100} z+b b_{000} x^{2} / a-\left(b_{000}+b_{200}\right) x y+\left(b b_{000} / a-\left(c_{000}+c_{200}\right)\right) x z \\
& -b\left(b_{000}+b_{200}\right) y^{2} / a-\left(b_{101}+c_{110}\right) y z+\left(b b_{000} / a-c_{101}\right) z^{2}, \\
\dot{y}= & b_{000}+a c_{100} z / b+b_{200} x^{2}+b\left(b_{000}+b_{200}\right) x y / a+b_{101} x z-b_{000} y^{2} \\
& +\left(\left(a^{2} b_{101}+b^{2} b_{101}+a^{2} c_{110}-a b c_{200}\right) /(a b)-c_{000}\right) y z+\left(\left(a c_{101}\right) / b-b_{000}\right) z^{2}, \\
\dot{z}= & c_{000}+c_{100} x-a c_{100} y / b+c_{200} x^{2}+c_{110} x y+c_{101} x z \\
& -\left(a^{2} b_{101}+b^{2} b_{101}+a^{2} c_{110}-a b c_{200}\right) y^{2} /(a b)-a c_{101} y z / b-c_{000} z^{2},
\end{aligned}
$$

having the invariant plane $a x+b y=0$ with $a b \neq 0$, and at most it has another invariant plane of the form either $x=0$ or $y=0$, because its extactic polynomial is

$\left((a x+b y)\left(a z\left(c_{100}+\left(b_{101}+c_{110}\right) y+c_{101} z\right)+b\left(b_{000}+b_{200}\left(x^{2}+y^{2}\right)+b_{101} x z-b_{000} z^{2}\right)\right)\right) /(a b)$,

taking either $c_{100}=b_{101}+c_{110}=c_{101}=b_{000}=b_{200}=0$, or $c_{100}=c_{101}=b_{000}=$ $b_{200}=b_{101}=0$, respectively.

In summary we have proved that quadratic polynomial differential systems on the sphere $\mathbb{S}^{2}$ can have at most 2 invariant meridians, and that there are such kind of systems having 2 invariant meridians. 
In the next proposition we give a polynomial differential system on the sphere $\mathbb{S}^{2}$ where the upper bound for the maximum number of invariant parallels is reached.

Proposition 9. Consider the polynomial differential system

$$
\begin{aligned}
& \dot{x}_{2}=1-x-x^{2}-y^{2}+z^{2}, \\
& \dot{x}_{3}=-2 y z, \\
& \dot{x}_{1}=y,
\end{aligned}
$$

on the sphere $\mathbb{S}^{2}$ of degree $(2,2,1)$. For this system the upper bound $m_{3}=1$ for the number of invariant parallels provided in Theorem 7 is reached.

Proof. Let $\mathcal{X}$ be the vector field associated to system (5). Then

$$
\mathcal{X}\left(x^{2}+y^{2}+z^{2}-1\right)=-2 y\left(x^{2}+y^{2}+z^{2}-1\right) .
$$

So $\mathcal{X}$ is a vector field on the sphere $\mathbb{S}^{2}$. Clearly the plane $z=0$ is invariant by the flow of $\mathcal{X}$, and is the unique invariant plane of the form $z=$ constant. So the proposition is proved.

\section{ACKNOWLEDGEMENTS}

The first author is partially supported by a FEDER-MINECO grant MTM201677278-P, a MINECO grant MTM2013-40998-P, and an AGAUR grant 2014SGR568. The second author acknowledges a BITDEFENDER postdoctoral fellowship from the Institute of Mathematics Simion Stoilow of the Romanian Academy, Contract of Sponsorship No. 262/2016 as well as partial support from a grant of the Romanian National Authority for Scientific Research and Innovation, CNCSUEFISCDI, project number PN-II-RU-TE-2014-4-0657.

\section{REFERENCES}

[1] R.F.S. Andrade And A. Rauh, The Lorenz model and the method of Carleman embedding, Phys. Lett. A 82 (1981), 276-278.

[2] J.C. Artés, B. Grünbaum and J. Llibre, On the number of invariant straight lines for polynomial differential systems, Pacific J. of Math. 184 (1998), 207-230.

[3] T.C. Bountis, A. Ramani, B. Grammaticos and B. Dorizzi, On the complete and partial integrability of non-Hamiltonian systems, Phys. A 128 (1984), 268-288.

[4] T. Carleman, Application de la théorie des équations intégrales linéaires aux systémes d'équations différentielles non linéaires, Acta Mathematica 59 (1932), 63-87.

[5] F. Cantrijn and W. SARLet, Generalizations of Noether's theorem in classical mechanics, SIAM Rev. 23 (1981), 467-494.

[6] J. Chavarriga, J. Llibre and J. Sotomayor, Algebraic solutions for polynomial systems with emphasis in the quadratic case, Expositiones Math. 15 (1997), 161-173.

[7] C.J. Christopher, Invariant algebraic curves and conditions for a center, Proc. Roy. Soc. Edinburgh 124A (1994), 1209-1229.

[8] C.J. Christopher And J. Llibre, Algebraic aspects of integrability for polynomial systems, Qualit. Th. Dyn. Syst. 1 (1999), 71-95.

[9] C.J. Christopher AND J. Llibre, Integrability via invariant algebraic curves for planar polynomial differential systems, Ann. Diff. Eqs. 16 (2000), 5-19.

[10] C. Christopher, J. Llibre and J.V. Pereira, Multiplicity of invariant algebraic curves in polynomial vector fields, Pacific J. of Math. 229 (2007), 63-117.

[11] G. Darboux, Mémoire sur les équations différentielles algébriques du premier ordre et du premier degré (Mélanges), Bull. Sci. math. 2ème série 2 (1878), 60-96; 123-144; 151-200. 
[12] V.A. Dobrovol'skit, N.V. Lokot' And J.M. Strelcyn, Mikhail Nikolaevich Lagutinskii (1871-1915): un mathématicien méconnu, (French) [Mikhail Nikolaevich Lagutinskii (18711915): an unrecognized mathematician] Historia Math. 25 (1998), 245-264.

[13] F. Dumortier, J. Llibre And J.C. Artés, Qualitative theory of planar differential systems, UniversiText, Springer-Verlag, New York, 2006.

[14] H.J. Giacomini, C.E. Repetto And O.P. Zandron, Integrals of motion of three-dimensional non-Hamiltonian dynamical systems, J. Phys. A 24 (1991), 4567-4574.

[15] J. Hietarinta, Direct methods for the search of the second invariant, Phys. Rep. 147 (1987), $87-154$.

[16] J.P. Jounolou, Equations de Pfaff algébriques, Lectures Notes in Mathematics 708 , Springer-Verlag, New York/Berlin, 1979.

[17] P.D. LAx, Integrals of nonlinear equations of evolution and solitary waves, Commun. Pure Appl. Math. 21 (1968), 467-490.

[18] J. LliBRE, Integrability of polynomial differential systems, Handbook of Differential Equations, Ordinary Differential Equations, Eds. A. Cañada, P. Drabek and A. Fonda, Elsevier, 2004, 437-533 pp.

[19] J. LliBRE AND Y. Bolaños, Rational first integrals for polynomial vector fields on algebraic hypersurfaces of $\mathbb{R}^{n+1}$, Int. J. Bifurcation and Chaos 22 (2012), 1250270-11 pp.

[20] J. LliBre AND J.C. Medrado, On the invariant hyperplanes for d-dimensional polynomial vector fields, J. Phys. A: Math. Gen. 40 (2007), 8385-8391.

[21] J. Llibre AND C. PessoA, Homogeneous polynomial vector fields of degree 2 on the 2dimensional sphere, Extracta Math. 21 (2006), 167-190.

[22] J. Llibre AND X. Zhang, Darboux integrability of real polynomial vector fields on regular algebraic hypersurfaces, Rend. Circ. Mat. Palermo 51 (2002), 109-126.

[23] J. Llibre AND X. Zhang, Darboux Theory of Integrability in $\mathbb{C}^{n}$ taking into account the multiplicity, J. of Differential Equations 246 (2009), 541-551.

[24] J. Llibre and X. Zhang, Rational first integrals in the Darboux theory of integrability in $\mathbb{C}^{n}$, Bull. Sci. Math. 134 (2010), 189-195.

[25] J. Llibre AND X. Zhang, On the Darboux integrability of the polynomial differential systems, Qualit. Th. Dyn. Syst. 11 (2012), 129-144.

[26] J. LlibRe AND X. ZHANG, Darboux theory of integrability for polynomial vector fields in $\mathbb{R}^{n}$ taking into account the multiplicity at infinity, Bull. Sci. Math. 133 (2009), 765-778.

[27] P.J. Olver, Applications of Lie groups to differential equations, Springer, New York, 1986.

[28] J.V. Pereira, Integrabilidade de equaçoes diferenciais no plano complexo, Monografias del IMCA 25, Lima, Peru, 2002.

[29] H. Poincaré, Sur l'intégration des équations différentielles du premier ordre et du premier degré I and II, Rendiconti del Circolo Matematico di Palermo 5 (1891), 161-191; 11 (1897), $193-239$.

[30] M.J. Prelle ANd M.F. Singer, Elementary first integrals of differential equations, Trans. Amer. Math. Soc. 279 (1983), 613-636.

[31] D. SCHLOMIUK, Elementary first integrals and algebraic invariant curves of differential equations, Expositiones Math. 11 (1993), 433-454.

[32] D. Schlomiuk, Algebraic particular integrals, integrability and the problem of the center, Trans. Amer. Math. Soc. 338 (1993), 799-841.

[33] D. Schlomiuk, Algebraic and geometric aspects of the theory of polynomial vector fields, in Bifurcations and Periodic Orbits of Vector Fields, D. Schlomiuk (ed.), 1993, 429-467 pp.

[34] M.F. Singer, Liouvillian first integrals of differential equations, Trans. Amer. Math. Soc. 333 (1992), 673-688.

[35] J.M. Strelcyn And S. Wojciechowski, A method of finding integrals for three-dimensional dynamical systems, Phys. Lett. A 133 (1988), 207-212.

Jaume Llibre, Departament de Matemàtiques, Universitat Autònoma de Barcelona, 08193 Bellaterra, Barcelona, Spain

E-mail address: jllibre@mat.uab.cat 
Adrian C. Murza, Institute of Mathematics "Simion Stollow" of the Romanian Academy, Calea Griviţei 21, 010702 Bucharest, Romania

E-mail address: adrian_murza@hotmail.com 